\title{
Conditioning by Minimizing Accessibility
}

\author{
Konstantinos Georgatos \\ 1 Department of Mathematics and Computer Science, John Jay College, \\ City University of New York, 445 West 59th Street, New York, NY 10019, \\ United States of America \\ 2 The Graduate Center, City University of New York, 365 Fifth Avenue, New York, \\ NY 10016, United States of America \\ kgeorgatos@jjay.cuny.edu
}

\begin{abstract}
This paper presents an axiomatization of a class of set-theoretic conditional operators using minimization of the geodesic distance defined as the shortest path generated by the accessibility relation on a frame. The objective of this modeling is to define conditioning based on a notion of similarity generated by degrees of indistinguishability.
\end{abstract}

\section{Introduction}

Stalnaker 27] developed semantics for counterfactual conditionals by using the following principle

$A>B$ is true at a state $s$ iff the closest $A$-world to $s$ is a $B$-world.

By "closest" one understands the most similar; closeness is identified with similarity. For example, one may reason that the counterfactual:

If it had rained, then I would have arrived later

is true because in the most similar world to the present where it had rained, I would have driven more carefully, and thus more slowly. However, it is a curious fact that neither Stalnaker nor Lewis, who relaxed the uniqueness assumption of the closest world [20, considered a standard notion of closeness because closeness is not judged with a global criterion but rather it is parameterized by worlds. Stalnaker made use of selection functions $f(x, A)$ where the variable $x$ is a world in the model, while Lewis made use of a class of closeness orderings $\leq_{x}$, one for each world $x$. Although such modelings are justifiable in theory, in practice similarity is modeled with global criteria. In Information Retrieval, for example, documents may be thought of as vectors in a metric space. The closer in space the more similar those documents are 25. Similarly, a popular global measure for similarity among propositional valuations is the number of variab! les they differ, their hamming distance (see [4]). Note that assuming a global distance does not exclude parameterization. In fact, a single global distance $d$ generates a closeness ordering $\leq_{x}$ for each epistemic state $x: y \leq_{x} z$ defined by $d(x, y) \leq d(x, z)$. Therefore, one wonders why global distance semantics for conditionals have not been studied or developed further. This is even more surprising in view of [26],

G. Bonanno, B. Löwe, and W. van der Hoek (Eds.): LOFT 2008, LNAI 6006, pp. 20-33, 2010.
(c) Springer-Verlag Berlin Heidelberg 2010 


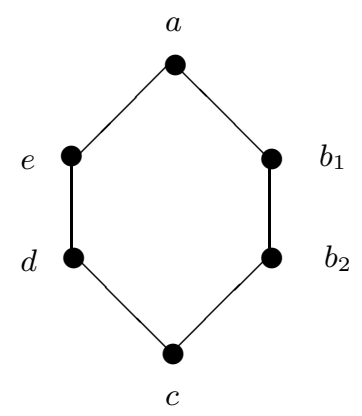

Fig. 1.

where it is shown that, for each parameterized ordering model, we can always find an equivalent model based on a global distance. In other words, we gain no advantage by parameterizing because an invariant distance relation can encode any set of parameterized orderings.

The purpose of the present paper is to introduce and characterize a class of conditional operators that are modeled by a global distance. We consider frames, that is, pairs $(T, R)$ where $T$ is a set of states and $R$ is an accessibility relation. Every frame comes equipped with a distance map called geodesic which is simply defined as the times we need to apply $R$ to reach a state. Minimization over a geodesic is straightforward. We illustrate the process with the following example where the accessibility relation $R$, represented by the edges of the graph, is reflexive and symmetric, in Figure 1. Let $A=\left\{a, b_{1}, b_{2}\right\}$, and $B=\left\{b_{1}, b_{2}\right\}$. We denote conditioning with $>$. Then $A>B=\left\{b_{2}, c\right\}$ because the closest $A$-worlds to $b_{2}$ and $c$ are $\left\{b_{1}, b_{2}\right\}$ and $\left\{b_{2}\right\}$ respectively. However, $d \notin A>B$ because its closest $A$-worlds are $\left\{a, b_{2}\right\}$. Similarly, we have $A>\neg B=\{e\}$.

We use the accessibility relation to represent indistinguishability. This idea is not new. Indeed, this is the idea behind the use of frames as semantics for epistemic modal logics. When a possible world $w_{2}$ is accessible from a word $w_{1}$ means that, for an agent in $w_{1}$, the world $w_{2}$ is (epistemically) indistinguishable from $w_{1}$. What are the properties of accessibility as indistinguishability? Many authors have argued that indistinguishability is better expressed through equivalence [117/5]; many have also dropped transitivity as early as [24] (see also [13] for similarity), while others have weakened transitivity, the most notable example being the $t$-norm transitivity of the similarity relation in Fuzzy sets [28. A set equipped with a reflexive symmetric relation has been called a proximity space in [2]. In the framework of Rough Sets [23, a reflexive, symmetric, and transitive relation of indistinguishability is called indiscernibility, and if it is only reflexive and symmetric it is called a tolerance relation [2921. In addition, their logical status has been well studied and give rise to orthologic [12], and the modal logic system $B$ (see [18]). Further, in the usual modal logics of belief reflexivity is dropped, as the agent may distinguish her present state from the one she believes (falsely) that she is in. We shall make no assumptions on 
indistinguishability although we show that properties such as reflexivity and symmetry can be added.

Examples are easily generated, as the accessibility relation needs no quantitative information. Metrics is perhaps the easiest way to generate indistinguishability; for example, let $x R y$ if and only if $d(x, y)<e$ for some appropriate fixed metric $d$ and non-negative real number $e$. Other examples follow:

Example 1. Let $T$ be the set of finite binary strings of finite length $n$. We can say that two strings are indistinguishable when they have the same length and differ in at most one digit. Otherwise, they are distinguishable.

Example 2. Let $T$ be a set of documents (sets of terms) and $n$ a positive integer. Two documents $d_{1}$ and $d_{2}$ are indistinguishable when they have at least $n$ common terms. That is,

$$
d_{1} R d_{2} \quad \text { iff } \quad\left|d_{1} \cap d_{2}\right| \geq n .
$$

The above examples are important and will be discussed in more detail in the next section.

We use the geodesic distance to express similarity. In [7], we argued that it is possible to view similarity (the geodesic distance) as a derived notion, the more basic concept being indistinguishability (the accessibility relation):

Our idea rests on the following maxim: two objects are similar when there is a context under which they are indistinguishable. Therefore, similarity can be measured with degrees of distinguishability. For example, although two similar houses might appear different in various details when we stand in front of them, they might appear identical if we observe them from an appropriate distance $x$. Thus, indistinguishability at distance $x$ implies similarity. The smaller the distance $x$, the less similar the objects are.

This way our approach follows closely the Stalnaker idea of conditioning by picking the most similar worlds [27. In our framework, the closest interpretations (according to the geodesic distance) are the most similar, as long as similarity is measured by degrees of distinguishability.

First, we discuss frames and their geodesics and present examples. In Section 3. we define geodesic conditional operators that correspond to update, and present their axioms and the associated characterization result (Theorem 9). In the next section, similar results are presented for a conditional operator based on revision. The form of Ramsey tests that allow us to go back and forth between the conditional and update/revision operators is also discussed.

\section{$2 \quad$ Frames and Their Geodesics}

In the following, we shall consider frames with a single accessibility relation, that is, pairs $F=(T, R)$ where $T$ is a set of states and $R \subseteq T \times T$ an accessibility 
relation between them. We shall write $x R y$ when $(x, y) \in R$. Given such a pair $F$ we can define its geodesic distance $d_{F}$, or simply $d$, as a map from $T \times T$ to $N \cup\{\infty\}$, where $N=\{1,2,3, \ldots\}$ is the set of natural numbers, defined by

$$
d_{F}(x, y)= \begin{cases}\min \left\{n \mid x R^{n} y\right\} & \text { if }\left\{n \mid x R^{n} y\right\} \neq \emptyset \\ \infty & \text { otherwise. }\end{cases}
$$

Note that $d_{F}$ is not a topological metric. First, its range does not include 0 . Although this could be mended, for example we could subtract 1 in the above definition, $d_{F}$ may fail identity. Nevertheless, if $R$ is assumed to be reflexive (see 311 and the range is extended to contain 0 , then $R$ is a pseudometric. If $R$ is connected, i.e., for all $x, y \in T$ there is $n \in N$ such that $x R^{n} y$, then the range of the geodesic restricts to $N$. In addition, (an extension of) the triangle inequality holds. In particular, we have the following

Lemma 3. Let $F=(T, R)$. Then:

1. For all $x \in T, d_{F}(x, x)=1$ iff $R$ is reflexive

2. $R$ is symmetric iff $d_{F}(x, y)=d_{F}(y, x)$

3. If $R$ is transitive then $d_{F}(x, y)=1$ or $\infty$

4. $d_{F}\left(x, y^{\prime}\right) \leq d_{F}(x, y)+d_{F}\left(y, y^{\prime}\right)$ (assuming $n+\infty=\infty+\infty=\infty$ )

5. For all $x, y \in T$ such that $d_{F}(x, y)=n$ with $2<n<\infty$ there is $z \in T$ with $z \neq x, y$ such that $d_{F}(x, y)=d_{F}(x, z)+d_{F}(z, y)$. In particular, we can choose $z$ so that $d_{F}(x, z)=1$.

We shall now discuss an interesting construction of a geodesic space given an arbitrary metric space. This construction is a straightforward modeling of the concept of threshold which was the original motivation of the present work. Let $(X, d)$ be a metric space and let $e>0$ be a real number representing a threshold. Then $\left(X, d_{e}\right)$ is called the $e$-threshold space of $(X, d)$, with $d_{e}(x, y)=d_{F}(x, y)$, where $F=(T, R)$ is a frame with $(x, y) \in R$ iff $d(x, y)<e$. Every $e$-threshold space is geodesic.

A more interesting space arises, if we let $d_{e}^{\mathrm{m}}(x, y)=n$, where $n=\min \{k$ : $d(x, y) \leq k e, k>1\}$. Call $\left(X, d_{e}^{\mathrm{m}}\right)$ the multiple $e$-threshold space of $(X, d)$. Unfortunately, we have $d_{e}^{\mathrm{m}}(x, y) \neq d_{F}(x, y)$, that is the multiple $e$-threshold space is not geodesic although $d_{e}^{\mathrm{m}}(x, y)$ is an integer valued map. However, an arbitrary integer distance map can be embedded into a geodesic space if we add enough elements as the following lemma shows.

Lemma 4. Let $(X, d)$ where $d$ is a map from $X \times X$ to $N \cup\{\infty\}$ satisfying the extended triangle inequality (as in Lemma 34). Then there exists a frame $F=(X, R)$ such that $d_{F}=d$.

Proof. Let $X^{\prime}=X \cup Y$, where

$$
Y=\left\{y_{\left(\left(x, x^{\prime}\right), k\right)}: d\left(x, x^{\prime}\right)=n<\infty, \text { and } k=1,2, \ldots, n-1\right\},
$$

that is, we add $n-1$ elements for all $x, x^{\prime} \in X$ such that $d\left(x, x^{\prime}\right)=n$. Also, let $R$ be defined by 
$-x R x^{\prime}$ iff $d\left(x, x^{\prime}\right)=1$,

- $x R y_{\left(\left\{x, x^{\prime}\right\}, 1\right)}$

- $y_{\left(\left\{x, x^{\prime}\right\}, n-1\right)} R x^{\prime}$,

- $y_{\left(\left\{x, x^{\prime}\right\}, m\right)} R y_{\left(\left\{x, x^{\prime}\right\}, m+1\right)}$, where $0<m<n-1$.

Consider the frame $F=\left(X^{\prime}, R\right)$. Observe that $d_{F}\left(x, x^{\prime}\right) \leq d\left(x, x^{\prime}\right)$, for $d\left(x, x^{\prime}\right)=$ $n<\infty$ implies the existence of sequence of $R$-related elements of $Y$ of lenth $n-1$. We shall show that $d\left(x, x^{\prime}\right)=d_{F}\left(x, x^{\prime}\right)$ by induction on $d_{F}\left(x, x^{\prime}\right)$.

Let $d_{F}\left(x, x^{\prime}\right)=1$. Then $x R x^{\prime}$ so by the above definition $d\left(x, x^{\prime}\right)=1$.

Assume $d\left(x, x^{\prime}\right)=d_{F}\left(x, x^{\prime}\right)$ for all $x, x^{\prime} \in X$ such that $d_{F}\left(x, x^{\prime}\right)=m$ with $m<n$.

Now, suppose $d_{F}\left(x, x^{\prime}\right)=n$. This means there is a $(n-1)$-length sequence of $R$-related elements between $x, x^{\prime}$. If this sequence contains no $x^{\prime \prime} \in X$ then the elements are all of the form $y_{\left(\left\{x, x^{\prime}\right\}, k\right)}$ and necessarily $d\left(x, x^{\prime}\right)=n$ by the definition of $Y$. If this sequence contains $x^{\prime \prime} \in X$, then $d_{F}\left(x, x^{\prime \prime}\right)+d_{F}\left(x^{\prime \prime}, x^{\prime}\right) \leq n$. By induction hypothesis, as $1<d_{F}\left(x, x^{\prime \prime}\right), d_{F}\left(x^{\prime \prime}, x^{\prime}\right)<n, d_{F}\left(x, x^{\prime \prime}\right)=d\left(x, x^{\prime \prime}\right)$ and $d_{F}\left(x^{\prime \prime}, x^{\prime}\right)=d\left(x^{\prime \prime}, x^{\prime}\right)$, and therefore $d\left(x, x^{\prime \prime}\right)+d\left(x^{\prime \prime}, x^{\prime}\right) \leq n$. By the triangle inequality $d\left(x, x^{\prime}\right) \leq n$. Since $n=d_{F}\left(x, x^{\prime}\right) \leq d\left(x, x^{\prime}\right)$, we have $d\left(x, x^{\prime}\right)=n$.

Finally, suppose $d_{F}\left(x, x^{\prime}\right)=\infty$, then $d\left(x, x^{\prime}\right)=\infty$ as $d_{F}\left(x, x^{\prime}\right) \leq d\left(x, x^{\prime}\right)$.

\section{$3 \quad$ Geodesic Conditioning}

Not surprisingly, conditional reasoning has been linked to belief change very early 2226]19. Although the relation between conditionals and the operators of update and revision is straightforward when the conditionals are not nested, there are results that extend this link to full conditional logics (see [14]16 for update, and [31011] for revision). Our approach is set theoretic and the results of this paper do not compare directly with these works. Nevertheless, the conditional operators we present should be viewed as an extension of those conditional logic systems to geodesic spaces.

We follow the presentation of conditioning of [15]. Halpern observed that an alternative to the usual syntax-semantics approach is to consider modalities and conditionals as set-theoretic operators. The justification is that eventually we interpret those operators set-theoretically, so there is no harm in doing this from the start. Mathematicians and economists have used this approach for a long time, the former by using probability theory, the latter by using events. The main advantage of this approach is that it gives easier completeness theorems because we skip limiting cases. Additionally, it is still possible to turn such completeness theorems into usual logic completeness theorems in a uniform way (see 15] for details).

Given a frame, there is a straightforward notion of conditioning based on its geodesic. As soon as we have a notion of distance, then we can choose the closest states. Let

$$
f_{F}(x, A)= \begin{cases}\left\{y \in A \mid d_{F}(x, y)=d_{F}(x, A)\right\} & d_{F}(x, A) \neq \infty \\ \emptyset & \text { otherwise }\end{cases}
$$

where $x \in T$ and $A \subseteq T$. 
Observe that $f_{F}$ is a selection function in the sense of Lewis, that is, it returns a subset of states rather than a unique state. Also note that $f_{F}$ extends to an operation between subsets with

$$
B \bullet A=\bigcup_{x \in B} f_{F}(x, A),
$$

where $A, B \subseteq T$. In [8], $B \bullet A$ was called the geodesic update operation.

Definition 5. Given a frame $F=(T, R)$ and $x \in T, A, B \subseteq T$ then we can define the geodesic conditional operator $>_{d}: 2^{T} \times 2^{T} \rightarrow 2^{T}$ by

$$
A>_{d} B=\left\{x: f_{F}(x, A) \subseteq B\right\} .
$$

The definition of $A>_{d} B$ readily translates to the following

$$
C \subseteq A>_{d} B \quad \text { iff } \quad C \bullet A \subseteq B
$$

which is a form of the Ramsey Test for the geodesic update operation. The fact that conditioning is defined through the Ramsey Test should not come as a surprise. As early as [14, it is known that the update is compatible with the Ramsey test. We shall attempt to formulate a similar identity for revision in the next section.

The axioms that characterize the geodesic conditional operator appear in Table 1. It is not difficult to show that $>_{d}$ satisfies all axioms apart from REFL and SYMM. Axiom NORM has appeared in [15] while ID, CV, MP, CN are straightforward translations of conditional logic axioms (for example see [22]). Axioms INDB, IND and $\mathrm{CN}^{*}$ axiomatize the behavior of $T>A$ that acts as the belief modality. Axioms INDB controls the base case, IND the induction case, while $\mathrm{CN}^{*}$ the iteration limit case. Axioms REFL and SYMM stipulate that the accessibility relation is reflexive and symmetric, respectively, and were not assumed for the results of this section.

Now, let a GC space be a pair $(T,>)$, where $T$ is a set of worlds and $>$ an operator on the subsets of $T$, that is, $>: 2^{T} \times 2^{T} \rightarrow 2^{T}$, which satisfies the axioms of Table 1. A GC space gives rise to a frame $(T, R)$, where $R$ is defined by

$$
x R y \quad \text { iff } \quad x \in T>A \Rightarrow y \in A
$$

for all $x, y \in T$ and $A \subseteq T$. Denote its associated geodesic conditional operator with $>_{d}$.

Lemma 6. Given a $G C$ space $(T,>)$ then, for all $A \subseteq T$ we have

$$
T>A=T>{ }_{d} A .
$$

Proof. First, suppose $x \in T>A$. Let $y \in T$ be such that $x R y$. We need to show that $y \in A$ but this is immediate from the definition of $R$. 
Table 1. System GC

$$
\begin{array}{cl}
\bigcap_{j \in J}\left(A>B_{j}\right)=A>\cap B_{j} & \text { NORM } \\
A>A=T & \text { ID } \\
\neg(A>\neg B) \cap(A>C) \subseteq(A \cap B)>C & \text { CV } \\
(A>B) \subseteq(T>(A \rightarrow B)) & \text { MP }
\end{array}
$$$$
\text { If } C \subseteq \neg(T>\neg A) \text {, then }(T>A \rightarrow B) \cap C=(A>B) \cap C \quad \text { INDB }
$$

If $C \subseteq T>\neg A$, then $(\neg(T>\neg A)>(T>A \rightarrow B)) \cap C=(A>B) \cap C$ IND

$$
\begin{aligned}
& \text { If } A \subseteq B \text {, then } B>\emptyset \subseteq A>\emptyset \quad \text { CN } \\
& \text { If } A \subseteq T>A \text {, then } A \subseteq \neg A>\emptyset \quad \mathrm{CN}^{*} \\
& T>A \subseteq A \quad \text { REFL } \\
& \neg A \subseteq T>\neg(T>A) \quad \text { SYMM }
\end{aligned}
$$

Next, suppose $x \in T>_{d} A$. Suppose towards a contradiction that $x \notin T>A$ and

$$
\neg A \cap \bigcap_{x \in T>{ }_{d} B} B=\emptyset
$$

which implies

$$
\bigcap_{x \in T>{ }_{d} B} B \subseteq A
$$

Using NORM we have

$$
T>\bigcap_{x \in T>{ }_{d} B} B \subseteq T>A .
$$

Applying again NORM we get

$$
\bigcap_{x \in T>{ }_{d} B} T>B \subseteq T>A .
$$

This shows that $x \in T>B$ which is a contradiction. Therefore,

$$
\neg A \cap \bigcap_{x \in T>{ }_{d} B} B \neq \emptyset
$$

and let $z \in T$ belong to the above set. Then we have that $x R z$ and $z \in \neg B$ which contradicts the initial hypothesis.

The following two lemmas are useful for the main result

Lemma 7. For all $x \in T, A \subseteq T$ and $n>1$,

$$
\left.d_{F}(x, A)=n \quad \text { iff } \quad d_{F}\left(x, \neg\left(T>_{d} \neg A\right)\right)\right)=n-1 .
$$


Proof. Suppose that $d_{F}\left(x, \neg\left(T>_{d} \neg A\right)\right)=n-1$. Then there exists $y \in \neg\left(T>_{d}\right.$ $\neg A)$ and $z \in A$ such that $d_{F}(x, y)=n-1$ and $d_{F}(y, z)=1$. By Lemma 344, we have $d_{F}(x, z) \leq n$. Therefore, $d_{F}(x, A) \leq n$. Suppose towards a contradiction that $d_{F}(x, A)=k<n$. Then there exists $w \in A$ such that $d_{F}(x, w)=k$. By Lemma 35, there exists $y \in T$ such that $d_{F}(x, y)=k-1$ and $d_{F}(y, w)=1$. The latter implies that $y \in \neg\left(T>_{d} \neg A\right)$ and therefore, $d_{F}\left(x, \neg\left(T>_{d} \neg A\right)\right)=k-1<$ $n-1$, a contradiction. The other direction is similar.

For the following, let $x^{*}=\neg \bigcup_{x \in A>_{d} \emptyset} A$, that is the set of all states accessible from $x$.

Lemma 8. For all $x \in T$ we have

$$
x^{*} \subseteq x^{*}>\emptyset .
$$

Proof. Suppose $y \in x^{*}$, so there is $n<\infty$ such that $x R^{n} y$. Let $y R z$ for a $z \in T$. Obviously $x R^{n+1} z$ and therefore $z \in x^{*}$. This shows that $y \in T>_{d} x^{*}$ so by Lemma 6 $y \in T>x^{*}$. Therefore, $x^{*} \subseteq T>x^{*}$. By $\mathrm{CN}^{*}$, we have $y \in x^{*}>\emptyset$.

The main result of this section is the following

Theorem 9. Given a $G C$ space, then, for all $A, B \subseteq T$

$$
A>B=A>_{d} B \text {. }
$$

Proof. We shall show that for all $x \in T$ and $A, B \subseteq T$,

$$
x \in A>B \quad \text { iff } \quad x \in A>{ }_{d} B,
$$

by induction of the distance of $x$ from $A$.

- Let $d(x, A)=1$.

Suppose $x \in A>B$. Let $y \in f_{F}(x, A)$. This implies that $x R y$. By MP, we have that $x \in T>A \rightarrow B$, so by the definition of $R$ we have that $y \in A \rightarrow B$. Since $y \in A$, we have $y \in B$. This shows that $x \in A>_{d} B$.

For the other direction, suppose $x \in A>_{d} B$. This implies $x \in T>_{d}$ $A \rightarrow B$ so, by Lemma 6 , we have $x \in T>A \rightarrow B$. Observe that $d(x, A)=1$ implies that $x \in \neg(T>\neg A)$. Applying INDB we have $x \in A>B$.

- Suppose the hypothesis is true for $d(x, A) \leq n$ and assume $d(x, A)=n+1$. Observe that since $d(x, A)>1$, we have $x \in T>_{d} \neg A$ and, by Lemma 6 . $x \in T>\neg A$.

Now, suppose $x \in A>B$ which is equivalent to

$$
x \in \neg(T>A)>(T>A \rightarrow B)
$$

given that $x \in T>\neg A$ and using IND. Observe that

$$
\neg(T>A)>(T>A \rightarrow B)=\neg\left(T>_{d} A\right)>\left(T>_{d} A \rightarrow B\right)
$$


using Lemma 6] We have that $d(x, \neg(T>\neg A))=n$ using Lemmas 7 and 6 ] By induction hypothesis

$$
\neg(T>A)>(T>A \rightarrow B)=\neg\left(T>_{d} A\right)>_{d}\left(T>_{d} A \rightarrow B\right) .
$$

So $x \in A>B$ is equivalent to $x \in \neg\left(T>_{d} A\right)>_{d}\left(T>_{d} A \rightarrow B\right)$ which is equivalent $x \in A>_{d} B$, given that $x \in T>_{d} \neg A$ and using IND.

- Finally, let $d(x, A)=\infty$. In this case, $f_{F}(x, A)=\emptyset$ so $x \in A>_{d} \emptyset$ and $x \in$ $A>_{d} B$ for all $B \subseteq T$. We have $A \subseteq \neg x^{*}$. By CN we get $\neg x^{*}>\emptyset \subseteq A>\emptyset$. By Lemma $8 x^{*} \subseteq A>\emptyset$. So $x \in A>\emptyset$, and therefore $x \in A>B$, for all $B \subseteq T$.

\section{Geodesic Conditioning Using Revision}

Recall that the selection function we used in the previous section could be extended to a selection function between two subsets which was subsequently used to define the update operator. There is an alternative way to extend the selection function $f_{F}$ to pairs of subsets: instead of pointwise minimization defined through union, we use global minimization. Let

$$
A * B= \begin{cases}\{x \in B: d(A, x)=d(A, B)\} & d(A, B)<\infty \\ \emptyset & \text { otherwise }\end{cases}
$$

As in the case of update, we called $A * B$ the (non-centered) geodesic revision of $A$ with $B$ in 9 . Observe that the second part of the definition could be eliminated for an alternative definition of the revision operator that does not satisfy consistency preservation.

Definition 10. Given a frame $F=(T, R)$ and $A, B \subseteq T$ then we can define the revision geodesic conditional operator $>_{d}^{*}: 2^{T} \times 2^{T} \rightarrow 2^{2^{T}}$ by

$$
A>_{d}^{*} B=\{C: C * A \subseteq B\} .
$$

Again as in the case of update, the above definition translates to the following Ramsey test:

$$
A * B \subseteq C \quad \text { iff } \quad A \in B>_{d}^{*} C \quad \mathrm{RMT}
$$

which is akin to the Ramsey test of the previous section in the sense that RMT forces conditioning to operate on a metalevel, that is, conditioning returns a set of subsets rather than a single subset. This difference should not be understood as a different mechanism. Indeed, we essentially minimize the induced accesibility $R^{*}$ relation on subsets defined by $A R^{*} B$ if $B=\{y: x R y, x \in A\}$. Since the induced accesibility is a function, this opens up the possibility of a simpler representation of the revision conditional based on the previous section. For example, consider the graph of Figure 2. Let $C=\left\{c_{1}, c_{2}\right\}$ and $D=\left\{c_{1}\right\}$. Then $C>_{d}^{*} D$ contains all the subsets whose distance from $c_{1}$ is (strictly) less than the distance from $c_{2}$. Those are $\left\{b_{1}\right\},\left\{b_{1}, a\right\},\left\{b_{1}, c_{2}\right\},\left\{c_{2}\right\}$, and $\left\{c_{2}, a\right\}$. 


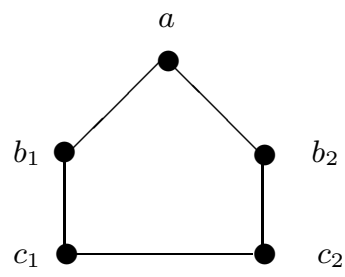

Fig. 2.

Table 2. System GCR

$$
\begin{array}{cl}
\bigcap_{j \in J}\left(A>B_{j}\right)=A>\bigcap_{j \in J} B_{j} & \text { NORM } \\
A>A=\mathcal{P}(T) & \text { ID } \\
\neg(T>\neg A) \cap(T>A \rightarrow B)=A>B & \text { INDB }
\end{array}
$$

If $X \in T>\neg A$, then $X \in A>\neg B$ iff $X^{\circ} \in A>B$ IND

If $X \subseteq Y$, then $Y \in T>A$ implies $X \in T>A$ SB

$$
\text { If } A \subseteq B \text {, then } B>\emptyset \subseteq A>\emptyset \quad \text { CN1 }
$$

If $X \subseteq Y$, then $Y \in A>\emptyset$ implies $X \in A>\emptyset \quad$ CN2

$$
\begin{array}{cl}
\text { If } A \in T>A \text {, then } A \in \neg A>\emptyset & \text { CN3 } \\
\text { If } A \in T>B \text {, then } A \subseteq B & \text { REFL } \\
\text { If } A \in T>\neg B \text {, then } B \in T>\neg A & \text { SYMM }
\end{array}
$$

The set of axioms that characterizes this class are those of Table 2 excluding REFL and SYMM. Axioms REFL and SYMM stipulate that the accessibility relation is reflexive and symmetric, respectively, and, as in the previous section, were not assumed for the results of this section. Notice that Axioms INDB and IND control induction on the frame. Axiom INDB is stronger than the Axiom MP of the preceding section. Axiom SB stipulates that beliefs persist when there is more information about the present state, i.e., monotonicity of beliefs. Axioms CN1, CN2, and CN3 stipulate the maximal expansion of our beliefs.

We proceed as in the previous section. Call a pair $(T,>)$, a GCR space if $>$ is an operation on the subsets of $T$ that returns a set of subsets of $T$, that is, $>: 2^{T} \times 2^{T} \rightarrow 2^{2^{T}}$, which satisfies the axioms of Table 2 Similarly, a GCR space gives rise to a frame $(T, R)$, where $R$ is defined by

$$
x R y \quad \text { iff } \quad\{x\} \in T>A \Rightarrow y \in A
$$

for all $x, y \in T$ and $A \subseteq T$. Denote its associated geodesic conditional operator with $>_{d}^{*}$. 
As in the previous section, we shall also make use of the following sets. The first set is the set of states the agent believes she is in when she is in $A$ :

$$
A^{\circ}=\bigcap_{A \in T>_{d} B} B .
$$

The second set is the set of states accessible from $A$ :

$$
A^{*}=T-\bigcup_{A \in B>{ }_{d} \emptyset} B .
$$

Lemma 11. If $(T,>)$ is a $G C R$ space, then for all $A \subseteq T$ we have $A \in T>A^{\circ}$.

Proof. Axiom NORM implies

$$
A \in \bigcap_{A \in T>B}(T>B)=T>\bigcap_{A \in T>B} B=T>A^{\circ} .
$$

Lemma 12. Given a GCR space $(T,>)$ then, for all $A \subseteq T$ we have

$$
T>A=T>_{d}^{*} A .
$$

Proof. First, suppose $C \in T>A$. We shall show that $C \in T>_{d}^{*} A$. By the definition of $>_{d}^{*}$, it is enough to show that $C * T \subseteq A$. Let $y \in C * T$ then by the definition of $*$ we have $d(C, y)=d(C, T)=1$ and, therefore, there exists $x \in C$ such that $d(x, y)=1$, i.e. $x R y$, which, by the definition of $R$ implies that if $\{x\} \in T>A$ then $y \in A$. Notice that $C \in T>A$ and therefore $\{x\} \in T>A$ by Axiom SB. So, we have $y \in A$.

For the opposite inclusion, suppose $C \in T>_{d}^{*} A$. If $\neg A \cap C^{\circ} \neq \emptyset$ then there exists $x \in C$ and $y \in \neg A$ such that $x R y$ which is a contradiction to our assumption. If $\neg A \cap C^{\circ}=\emptyset$ then $C^{\circ} \subseteq A$, so $T>C^{\circ} \subseteq T>A$ by Axiom NORM. So by Lemma 11$] \subseteq T>A$.

Observe that the above Lemma implies

$$
A^{\circ}=\bigcap_{A \in T>{ }_{d}^{*} B} B=\bigcap_{A \in T>B} B,
$$

that allow us to make use of the operator $-{ }^{*}$ in the axioms.

We omit the proof of the following lemma which is similar to the proof Lemma 7.

Lemma 13. For all $A, B \subseteq T$ and $n>1$,

$$
d_{F}(A, B)=n \quad \text { iff } \quad d_{F}\left(A^{\circ}, B\right)=n-1 .
$$


We have the following

Lemma 14. For all $A \subseteq T$ we have $C^{*} \in T>C^{*}$.

Proof. Suppose $C^{*} \notin T>C^{*}$ so $C^{*} \notin T>_{d}^{*} C^{*}$ by Lemma 12. Therefore $C^{*} * T \nsubseteq C^{*}$, which implies that there exist $x \in C^{*}$ and $y \in T$ such that $x R y$ and $y \in \neg C^{*}$, which contradicts that $d_{F}\left(C^{*}, \neg C^{*}\right)=\infty$.

We may now show the following theorem

Theorem 15. Given a GCR space, then, for all $A, B \subseteq T$

$$
A>B=A>_{d}^{*} B .
$$

Proof. We shall show that for all $C \subseteq T$ and $A, B \subseteq T$,

$$
C \in A>B \quad \text { iff } \quad C \in A>{ }_{d} B,
$$

by induction of the distance of $C$ from $A$.

- Let $d(C, A)=1$.

Suppose $C \in A>B$. So $C \in T>A \rightarrow B$, so $C \in T>A>{ }_{d}^{*} B$ by Lemma 12. Therefore $C * A \subseteq A \rightarrow B$, so $C * A \subseteq B$ and so $C \in A>_{d}^{*} B$.

For the other direction, suppose $C \in A>_{d} B$. This implies that $C \in$ $T>{ }_{d} A \rightarrow B$ by Axiom MP, so by Lemma 12 we have $C \in T>A \rightarrow B$. We have that $d(C, A)=1$ implies that $C \in \neg\left(T>_{d}^{*} \neg A\right)$ and again by Lemma 12 we get $C \in \neg(T>\neg A)$. Applying Axiom MP we have that $C \in A>B$.

- Suppose the hypothesis is true for $d(C, A) \leq n$ and let $d(x, A)=n+1$.

Observe that since $d(C, A)>1$ then we have $C \in T>_{d} \neg A$ and, by Lemma 12, $C \in T>\neg A$.

Suppose $C \in A>B$ which is equivalent to $C^{\circ} \in A>B$ by Axiom IND. By Lemma 13, we $d\left(C^{\circ}, A\right) \leq n$ and therefore we can apply the induction hypothesis to infer that $C^{\circ}>A=C^{\circ}>_{d}^{*} A$. Again by applying Axiom IND we have $C \in A>_{d}^{*} B$.

- Finally, let $d(C, A)=\infty$. We have $C \in A>_{d}^{*} \infty$ and we shall show $C \in A>$ $\emptyset$. By Lemma 14, we have $C^{*} \in T>C^{*}$ which implies $C^{*} \in \neg C^{*}>\emptyset$ using Axiom CN3. By Axiom CN2, we get $C^{*} \in A>\emptyset$, because $A \subseteq \neg C^{*}$ using Axiom CN1. Applying Axiom CN2, we get $C \in A>\emptyset$.

\section{Conclusion}

We introduced a class of set-theoretic conditional operators based on a global distance map of indistinguishability, and characterized this class by a finite set of axiom schemas. This result extends to a variety of properties we can assume for the indistinguishability relation, such as reflexivity and symmetry. These results belong to a larger study of the reasoning based on the minimization of a geodesic map. In particular, we have studied and characterized belief revision and update operators 98 . 
Although our approach is part of the wider field of distance-based reasoning, its most salient feature is that it provides a bridge between qualitative and quantitative reasoning. The reason for this is twofold: first, quantitative information prone to error is not continuous. The threshold of error limits the range of values into integers which in turn can be embedded into a geodesic space. Second, limited observational powers introduce vagueness phenomena in human reasoning and we have argued [7] that such phenomena can be modeled satisfactorily using a binary relation of indistinguishability. The use of indistinguishability may not be applicable to all facets of formal epistemology but it seems particularly suitable when a metric space is already in place.

\section{References}

1. Aumann, R.: Agreeing to Disagree. Ann. Stat. 4, 1236-1239 (1976)

2. Bell, J.L.: A New Approach to Quantum Logic. Brit. J. Philos. Sci. 37, 83-99 (1986)

3. Boutilier, C.: Revision Sequences and Nested Conditionals. In: Bajcsy, R. (ed.) IJCAI 1993, pp. 519-525. Morgan Kaufmann, California (1993)

4. Dalal, M.: Investigations into a Theory of Knowledge Base Bevision: Preliminary Report. In: Rosenbloom, P., Szolovits, P. (eds.) AAAI 1988, vol. 2, pp. 475-479. AAAI Press, Menlo Park (1988)

5. Fagin, R., Halpern, J.Y., Vardi, M.Y.: A model-theoretic Analysis of Knowledge. J. ACM 38(2), 382-428 (1991)

6. Gärdenfors, P.: Knowledge in Flux. MIT Press, Cambridge (1985)

7. Georgatos, K.: On Indistinguishability and Prototypes. Log. J. IGPL 11(5), 531545 (2003)

8. Georgatos, K.: Belief Update Using Graphs. In: Wilson, D., Lane, H.C. (eds.) FLAIRS 2008, pp. 649-654. AAAI Press, Menlo Park (2008)

9. Georgatos, K.: Geodesic Revision. J. Log. Comput. 19, 447-459 (2008)

10. Giordano, L., Gliozzi, V., Olivetti, N.: Iterated Belief Revision and Conditional Logic. Stud. Log. 70(1), 23-47 (2002)

11. Giordano, L., Gliozzi, V., Olivetti, N.: Weak AGM Postulates and Strong Ramsey Test: A Logical Formalization. Artif. Intell. 168(1-2), 1-37 (2005)

12. Goldblatt, R.I.: Semantic Analysis of Orthologic. J. Philos. Log. 3, 19-35 (1974)

13. Goodman, N.: The Structure of Appearance, 3rd edn. Boston Studies in the Philosphy of Science, vol. 53. Reidel, Dordrecht (1977)

14. Grahne, G.: Updates and Counterfactuals. In: Allen, J.F., Fikes, R., Sandewall, E. (eds.) KR 1991, pp. 269-276. Morgan Kaufmann, San Francisco (1991)

15. Halpern, J.Y.: Set-theoretic Completeness for Epistemic and Conditional Logic. Ann. Math. Artif. Intell. 26(1-4), 1-27 (1999)

16. Herzig, A.: Logics for Belief Base Updating. In: Gabbay, D., Smets, P. (eds.) Handbook of Defeasible Reasoning and Uncertainty Management, vol. 3, pp. 189-231. Kluwer Academic Publishers, Dordrecht (1998)

17. Hintikka, J.: Knowledge and Belief. Cornell University Press, Ithaca (1962)

18. Hughes, G.E., Cresswell, M.J.: A Companion to Modal Logic. Methuen, London (1984)

19. Katsuno, H., Satoh, D.: A Unified View of Consequence Relation, Belief Revision and Conditional Logic. In: Mylopolous, J., Reiter, R. (eds.) IJCAI 1991, pp. 406412. Morgan Kaufmann, San Francisco (1991) 
20. Lewis, D.: Counterfactuals. Harvard University Press, Cambridge (1973)

21. Nieminen, J.: Rough tolerance equality. Fund. Inform. 11, 288-294 (1988)

22. Nute, D.: Conditional Logic. In: Gabbay, D., Guenthner, F. (eds.) Handbook of Philosophical Logic, vol. II, pp. 387-439. Reidel, Dordrecht (1984)

23. Pawlak, Z.: Rough Sets - Theoretical Aspects of Reasoning about Data. Kluwer Academic Publishers, Dordrecht (1991)

24. Poincaré, H.: La Valeur de la Science. Flammarion, Paris (1905)

25. Salton, G.: Automatic Text Processing: The Transformation, Analysis, and Retrieval of Information by Computer. Addison-Wesley, Reading (1989)

26. Schlechta, K., Makinson, D.: Local and Global Metrics for the Semantics of Counterfactual Conditionals. J. Appl. Non-Class. Log. 4(2), 129-140 (1994)

27. Stalnaker, R.: A Theory of Conditionals. In: Rescher, N. (ed.) Studies in Logical Theory. American Philosophical Quarterly Monograph Series, vol. 52, pp. 98-112. Blackwell, Oxford (1968)

28. Zadeh, L.A.: Similarity Relations and Fuzzy Orderings. Inform. Sciences 3, 177-200 (1971)

29. Zeeman, E.C.: The Topology of the Brain and Visual Perception. In: Fort, M.K. (ed.) The Topology of 3-Manifolds, pp. 240-256. Prentice Hall, Englewood Cliffs (1962) 